\title{
Natural Products: New Hope for Nonalcoholic Steatohepatitis?
}

\author{
Michael B. Zemel
}

$\mathbf{N}$ ONALCOHOLIC FATTY LIVER DISEASE (NAFLD) is a spectrum of disorders characterized by hepatic lipid accumulation and cellular degeneration in the absence of significant alcohol consumption that may progress to nonalcoholic steatohepatitis (NASH), cirrhosis, or hepatocellular carcinoma (HCC). ${ }^{1,2}$ Owing in large part to the obesity epidemic, ${ }^{3}$ NAFLD is the most prevalent chronic liver disease and is projected to become the leading cause of liver transplantation. The rapidly growing NAFLD epidemic parallels the obesity trajectory and is estimated to be $25 \%$ in the general population, and increases to $\sim 75 \%$ to $90 \%$ in patients with obesity or diabetes. ${ }^{4}$ Although simple nonalcoholic fatty liver (NAFL) in the absence of hepatocellular injury is generally benign and nonprogressive, $\sim 30 \%$ of NAFL patients develop NASH that can further progress to cirrhosis, end-stage liver disease, and HCC. ${ }^{5,6}$ NAFLD patients exhibit increased morbidity and total mortality due to cardiovascular disease as well as liver-related complications compared with the general population. ${ }^{5}$

Although NASH is a major public health concern, there are currently no approved treatments. Recommended firstline therapy is lifestyle change, including physical activity and weight loss. Weight loss of $3 \%$ to $5 \%$, whether through hypocaloric diet, exercise, or both, improves hepatic steatosis but not other features of NASH, whereas more substantial weight loss $(\sim 7 \%)$ is required to regress NASH, and $10 \%$ to improve fibrosis ${ }^{3,7}$; however, compliance with the lifestyle changes necessary to accomplish and maintain weight loss is often challenging. 5,8 Other therapeutic approaches focus on controlling concurrent metabolic disorders such as diabetes and hyperlipidemia. ${ }^{9}$ Antidiabetic drugs (e.g., metformin and pioglitazone), lipid-lowering drugs (e.g., statins and fibrates), antiobesity medication (e.g., orlistat), and antioxidants (e.g., vitamin E) may improve some metabolic parameters; however, they either have minimal effects on liver histology or have other adverse effects limiting their use. ${ }^{8,10,11}$

Pharmacotherapy for NASH is under intense development, with $>40$ clinical programs underway. However, latestage programs have thus far shown marginal efficacy and have troubling safety signals, suggesting the need for alternative approaches. In this issue of the journal, Mizrahi et $a .^{12}$ provide promising data demonstrating antiinflammatory effects of Hoodia extracts (Hesperaloe parviflora), which result in marked improvements in chemically induced immune hepatitis. They further show that $H$. parviflora improves glucose tolerance as well as hepatic triglycerides and liver histology in both genetic (ob/ob mice) and nutritionally induced (high-energy diets) models of NAFLD/NASH. Enthusiasm is tempered by lack of safety data, as another member of the genus (Hoodia gordonii) was shown to cause short-term changes in vital signs and some laboratory parameters. ${ }^{13}$ However, given the diversity of chemical composition among Hoodia spp., comparable safety signals cannot be presumed to be present in $H$. parviflora. Thus, these findings are cause for optimism, although there is much to be done, including identifying the bioactive component(s) and optimizing dosage, extending these findings to a high-fidelity translational model of NASH inflammation fibrosis, such as the AMLN or DIAMOND mouse models ${ }^{14}$ and, of course, clinical translation.

\section{REFERENCES}

1. Festi D, Colecchia A, Sacco T, Bondi M, Roda E, Marchesini G: Hepatic steatosis in obese patients: Clinical aspects and prognostic significance. Obes Rev 2004;5:27-42.

2. Festi D, Schiumerini R, Marzi L, et al.: Review article: The diagnosis of non-alcoholic fatty liver disease-Availability and accuracy of non-invasive methods. Aliment Pharmacol Ther 2013;37:392-400.

3. Polyzos SA, Kountouras J, Mantzoros CS: Obesity and nonalcoholic fatty liver disease: From pathophysiology to therapeutics. Metabolism 2019;92:82-97.

4. Zeng L, Tang WJ, Yin JJ, Zhou BJ: Signal transductions and nonalcoholic fatty liver: A mini-review. Int J Clin Exp Med 2014;7:1624-1631.

5. Dietrich P, Hellerbrand C: Non-alcoholic fatty liver disease, obesity and the metabolic syndrome. Best Pract Res Clin Gastroenterol 2014;28:637-653.

6. Hashimoto E, Tokushige K, Ludwig J: Diagnosis and classification of non-alcoholic fatty liver disease and non-alcoholic steatohepatitis: Current concepts and remaining challenges. Hepatol Res 2014;45:20-28.

7. Chalasani N, Younossi Z, Lavine JE, et al.: The diagnosis and management of nonalcoholic fatty liver disease: Practice guidance from the American Association for the Study of Liver Diseases. Hepatology 2017;67:328-357.

8. Chalasani N, Younossi Z, Lavine JE, et al.: The diagnosis and management of non-alcoholic fatty liver disease: Practice Guideline by the American Association for the Study of Liver 
Diseases, American College of Gastroenterology, and the American Gastroenterological Association. Hepatology 2012;55: 2005-2023.

9. Ratziu V: Pharmacological agents for NASH. Nat Rev Gastroenterol Hepatol 2013;10:676-685.

10. Takaki A, Kawai D, Yamamoto K: Molecular mechanisms and new treatment strategies for non-alcoholic steatohepatitis (NASH). Int J Mol Sci 2014;15:7352-7379.

11. Ozturk ZA, Kadayifci A: Insulin sensitizers for the treatment of non-alcoholic fatty liver disease. World J Hepatol 2014;6:199-206.

12. Mizrahi M, Ben A, Adar T, Sklair ML, Gaska S, Ilan Y: Oral administration of Hoodia parviflora alleviates insulin resistance and nonalcoholic steatohepatitis. J Med Food 2019;00:1-10.
13. Blom WA, Abrahamse SL, Bradford R, et al: Effects of 15-d repeated consumption of Hoodia gordonii purified extract on safety, ad libitum energy intake, and body weight in healthy, overweight women: A randomized controlled trial. Am J Clin Nutr 2011;94:1171-1181.

14. Hansen HH, Feigh M, Veidal SS, Rigbolt KT, Vrang N, Fosgerau $\mathrm{K}$ : Mouse models of nonalcoholic steatohepatitis in preclinical drug development. Drug Discov Today 2017;22:1707-1718.

-Michael B. Zemel, PhD

Editor-in-Chief 